\title{
Transition Metal Impurities on the Bond-Centered Site in Germanium
}

\author{
S. Decoster, ${ }^{1}$ S. Cottenier, ${ }^{1,2,3}$ B. De Vries, ${ }^{1}$ H. Emmerich, ${ }^{2}$ U. Wahl, ${ }^{4}$ J. G. Correia, ${ }^{4}$ and A. Vantomme ${ }^{1}$ \\ ${ }^{1}$ Instituut voor Kern- en Stralingsfysica and INPAC, KULeuven, BE-3001 Leuven, Belgium \\ ${ }^{2}$ Computational Materials Engineering (CME), Institute for Minerals Engineering (GHI), Center for Computational Engineering \\ Science (CCES) \& Jülich-Aachen Research Alliance (JARA), RWTH Aachen University, DE-52064 Aachen, Germany \\ ${ }^{3}$ Center for Molecular Modeling, Ghent University, Proeftuinstraat 86, BE-9000 Ghent, Belgium \\ ${ }^{4}$ Instituto Tecnológico e Nuclear, UFA, Estrada Nacional 10, apartment 21, 2686-953 Sacavém, Portugal
}

(Received 10 October 2008; published 10 February 2009)

\begin{abstract}
We report on the lattice location of ion implanted $\mathrm{Fe}, \mathrm{Cu}$, and $\mathrm{Ag}$ impurities in germanium from a combined approach of emission channeling experiments and ab initio total energy calculations. Following common expectation, a fraction of these transition metals (TMs) was found on the substitutional Ge position. Less expected is the observation of a second fraction on the sixfold coordinated bond-centered site. Ab initio calculated heats of formation suggest this is the result of the trapping of a vacancy by a substitutional TM impurity, spontaneously forming an impurity-vacancy complex in the split-vacancy configuration. We also present an approach to displace the TM impurities from the electrically active substitutional site to the bond-centered site.
\end{abstract}

Compared to silicon, free charge carriers have a higher mobility and dopants have a lower activation temperature in Ge [1], which makes it an increasingly important and attractive material in metal-oxide semiconductor fieldeffect transistors [2]. Despite intensive research, several fundamental properties of this semiconductor are still poorly known. Transition metals (TMs) produce deep-level states in the semiconductor band gap and are hence detrimental for the electrical properties of integrated circuits, even in small concentrations [3]. Since the lattice site of TMs has a major influence on their electrical activity, lattice location studies are important. For Ge, the electrical behavior of ion implanted as well as in-diffused TM impurities has been studied quite extensively ([4-6] and references therein), but the information about their lattice location in Ge is rather puzzling. From electrical characterization, the TMs are found to act as multiple acceptors in $\mathrm{Ge}$, and in accordance with the simple valence model, most of the induced deep-level states in the band gap have been attributed to TMs on the substitutional $(S)$ site. However, the full picture seems to be more complicated. Mössbauer spectroscopy experiments after recoil implantation of ${ }^{57 m} \mathrm{Fe}$ and ion implantation of ${ }^{57} \mathrm{Mn}$ in Ge have revealed that the $\mathrm{Fe}$ atoms also partly occupy the tetrahedral interstitial $(T)$ site and a third site believed to be related to $\mathrm{Fe}_{i}-V$ complexes [7,8]. From emission channeling experiments similar to the ones presented here, a large fraction of ion implanted ${ }^{67} \mathrm{Cu}$ was found on the $S$ site, together with a smaller fraction located halfway between the $S$ and the bond-centered $(B C)$ site [9]. On the computational side, $a b$ initio calculations indicate that the $S$ site is favored over the $T$ site for $3 d$ transition metals in Ge as well as in $\mathrm{Si}$ $[10,11]$. Other theoretical studies in Ge focussed on impurity-vacancy complexes with impurities from the $s p$ series, with conflicting geometrical results $[12,13]$.
To clarify these puzzling experimental data, we present a direct lattice location study of $\mathrm{Fe}, \mathrm{Cu}$, and $\mathrm{Ag}$ in $\mathrm{Ge}$ with the emission channeling (EC) technique [14]. In an EC experiment, charged particles emitted from an implanted radioactive isotope are guided by the potential of atomic rows and planes while traveling through the crystal. The resulting anisotropic electron emission pattern around lowindex crystal directions is characteristic for the lattice site occupied by the emitting atom and is measured with a $2 \mathrm{D}$ energy- and position-sensitive Si detector of $22 \times 22$ pixels. The advantages of this technique are its high accuracy in comparison with regular ion channeling techniques and the use of very low implantation fluences which allows us to study isolated atoms.

The radioactive isotopes ${ }^{59} \mathrm{Mn},{ }^{67} \mathrm{Cu}$ and ${ }^{111} \mathrm{Ag}$ have been implanted at the ISOLDE facility in CERN to study the lattice location of $\mathrm{Fe}, \mathrm{Cu}$, and $\mathrm{Ag}$, respectively. ${ }^{59} \mathrm{Mn}$ (4.6 s) rapidly decays into the long-lived $\beta^{-}$-emitter ${ }^{59} \mathrm{Fe}$ (44.6 d), which decays to ${ }^{59} \mathrm{Co}$. The ${ }^{59} \mathrm{Fe}$ nucleus receives an average recoil of $200 \mathrm{eV}$ which assures the atom gets reimplanted and is not influenced by the lattice site of its precursor. ${ }^{67} \mathrm{Cu}(61.9 \mathrm{~h})$ decays into stable ${ }^{67} \mathrm{Zn}$ by emitting $\beta^{-}$-particles and in a similar way ${ }^{111} \mathrm{Ag}(7.45 \mathrm{~d})$ decays into stable ${ }^{111} \mathrm{Cd}$. The implantations have been performed at room temperature in undoped $\langle 111\rangle$-Ge with an energy of $60 \mathrm{keV}$ to fluences of $1.0 \times 10^{13} \mathrm{~cm}^{-2}$ for $\mathrm{Fe}, 6.6 \times$ $10^{12} \mathrm{~cm}^{-2}$ for $\mathrm{Cu}$, and $5.0 \times 10^{12} \mathrm{~cm}^{-2}$ for $\mathrm{Ag}$. To obtain accurate and unambiguous results, emission patterns along the four crystal directions $\langle 100\rangle,\langle 111\rangle,\langle 211\rangle$, and $\langle 110\rangle$ were measured, analyzed consistently and fitted to a set of simulated spectra. These simulations [15] have been performed for several high-symmetry sites such as the $S, T$, $B C$, hexagonal $(H)$ and the so-called $A B, S P, Y$, and $C$ sites [16], as well as for discrete displacements between these sites along the $\langle 111\rangle$-, $\langle 100\rangle$ - and $\langle 110\rangle$-direction. To moni- 
tor the thermal stability of the lattice location of the implanted impurities, the measurements were performed on as implanted samples, as well as after annealing up to $500{ }^{\circ} \mathrm{C}$ in vacuum $\left(<10^{-5}\right.$ mbar $)$ during $10 \mathrm{~min}$.

Figures 1(a)-1(d) show the normalized electron emission patterns around the $\langle 111\rangle,\langle 100\rangle,\langle 110\rangle$, and $\langle 211\rangle$-axes for ${ }^{111} \mathrm{Ag}$ in $\mathrm{Ge}$. In all directions, an increased normalized yield along the measured axis is visible (i.e., axial channeling), indicating that at least a fraction of the implanted $\mathrm{Ag}$ atoms will be located substitutionally. However, comparing the experimental patterns on panels (a) to (d) with the simulated patterns for purely substitutional ${ }^{111} \mathrm{Ag}$ atoms on, respectively, panels (e), (g), (i), and (k) reveals a number of discrepancies. The experimental pattern along the $\langle 110\rangle$-direction shows a split channeling peak in the center of panel (c) - which is not present in the simulation on panel (i) - while along the $\langle 100\rangle$-direction, the measured axial channeling effect on panel (b) is much less pronounced than the simulated one on panel $(\mathrm{g})$. More discrepancies can be found by investigating the measured planar channeling effects.

This visual analysis indicates that the $\mathrm{Ag}$ impurities occupy at least a second high-symmetry site. Therefore, a quantitative fitting procedure has been applied, allowing the $\mathrm{Ag}$ atoms to occupy up to three different sites. Adding either $T, H, S P, A B, Y$, or $C$ sites to the substitutional fraction results only in an insignificant fit improvement $\left(\chi^{2}\right.$ improvement $<2 \%$ ), while adding a fraction on the $B C$ site remarkably improves the fit, up to $25 \%$. Visual inspection of the simulations for the $S$ and $B C$ sites in Figs. 1(e)-1(1), shows that a linear combination of these simulations is able to solve the discrepancies described above, leading to the acceptably accurate fits in Figs. 1(m)-1(p). Adding more high-symmetry sites results in only marginal improvements of the fits (i.e., $\chi^{2}$ improvement $<2 \%$ ), allowing us to conclude that only small $\mathrm{Ag}$ fractions $(<3 \%)$ occupy other high-symmetry sites.

In the best fit to the experimental patterns, 21(3)\% of the implanted $\mathrm{Ag}$ atoms occupy the $S$ site and 33(4)\% the $B C$ site. The remaining fraction, which will be referred to as the random fraction, will be discussed in the next paragraph. After analyzing the patterns for $\mathrm{Fe}, \mathrm{Cu}$ and $\mathrm{Ag}$ as implanted and after several annealing stages, the general trend is clear (Fig. 2): the three TMs are partially found on the $S$ site and partially on the $B C$ site.

Heavy ion implantation produces highly damaged regions, especially in materials with small lattice binding energies such as Ge, even for the low fluences used in this study. This implantation damage has a double influence on the results shown here, and more specifically on the random fraction. First, due to the deterioration of the crystal structure, a fraction of the implanted radioactive isotopes will be located in damaged regions with reduced local crystallinity. Second, a fraction of the electrons emitted from an undamaged region will pass through damaged crystal regions, enhancing the probability for dechanneling - thus masking the impurity's lattice site. Both effects will result in an isotropic background to the patterns and consequently in the random fraction, observed in the experiments. This damage effect implies that the fractions

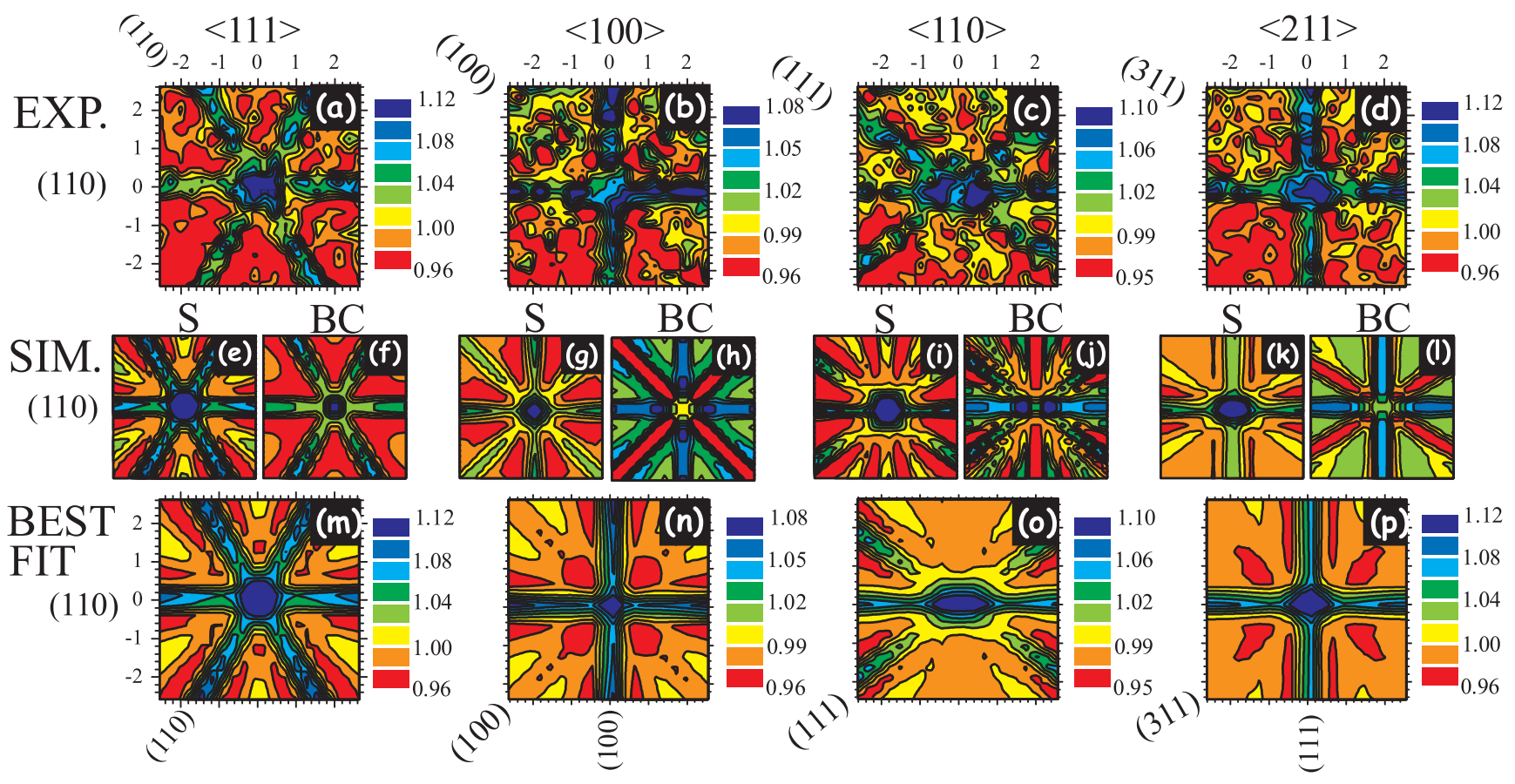

FIG. 1 (color online). (a)-(d) Two-dimensional electron patterns emitted from ${ }^{111} \mathrm{Ag}$ in Ge around the $\langle 111\rangle,\langle 100\rangle,\langle 110\rangle$, and $\langle 211\rangle$-axes, following a $400^{\circ} \mathrm{C}$ annealing step in vacuum; simulated patterns around the (e), (f) $\langle 111\rangle,(\mathrm{g}),(\mathrm{h})\langle 100\rangle,(\mathrm{i}),(\mathrm{j})\langle 110\rangle$ and (k), (1) $\langle 211\rangle$-axes for $\mathrm{Ag}$ on the $S$ site and on the $B C$ site, respectively; (m)-(p) the best fits to the experimental patterns. 


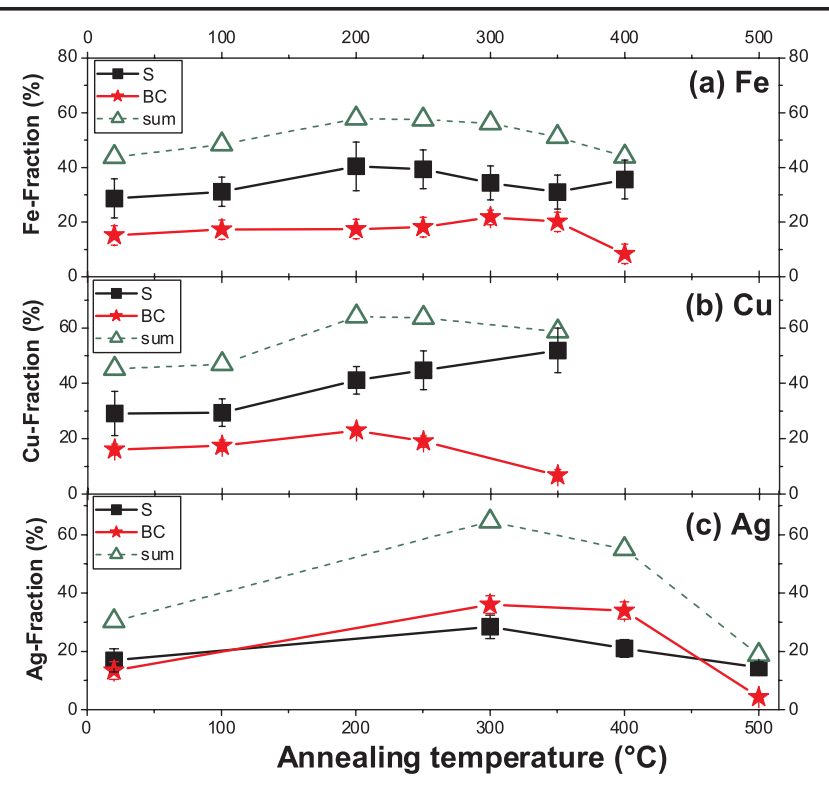

FIG. 2 (color online). Fraction of the implanted $\mathrm{Fe}(\mathrm{a}), \mathrm{Cu}(\mathrm{b})$, and $\mathrm{Ag}$ atoms (c) on the $S$ site ( $\square$ ) and the $B C$ site ( $\star$ ) in $\mathrm{Ge}$, together with the total fraction $\left(\triangle=\boldsymbol{\square}_{+} \star\right)$ on high-symmetry sites, as a function of annealing temperature.

presented in this work should be treated as lower limits to the real ones. The increasing fraction of TMs on highsymmetry sites after the first annealing steps (Fig. 2) indicates that the implantation damage is at least partly recovered. The drastic decrease of this fraction in the case of $\mathrm{Ag}$ [Fig. 2(c)] after annealing at $500{ }^{\circ} \mathrm{C}$ is related to the diffusion of $\mathrm{Ag}$ atoms and will not be discussed in more detail here.

In accordance with the studies discussed in the introduction [4-10], we found a large fraction of the TMs on the $S$ site in Ge after ion implantation. The prevalence of the $S$ site is consistent with theoretical work $[10,11]$ where it was found to be more favorable than the $T$ and $H$ sites. More intriguing is the occupation of the $B C$ site, for which we found no unambiguous experimental evidence in literature. In order to understand the existence of this $B C$ site, a complementary $a b$ initio study has been performed. We have calculated the heat of formation of 3 impurity sites in Ge: the $S$ site, the $T$ site, and the impurity on the $S$ site with one vacancy in the nearest neighbor shell $(S+V)$. The latter complex was taken into account because ion implantation produces a large amount of vacancies, which are mobile at room temperature in Ge [17] and might be trapped by impurities. The heats of formation reported in Table I are calculated according to

$$
\Delta H_{f}=E_{\text {sup }}^{\text {imp }}-\mu_{\text {imp }}-\left(32 E_{\text {sup }}^{\text {id }}-n \mu_{\mathrm{Ge}}\right)
$$

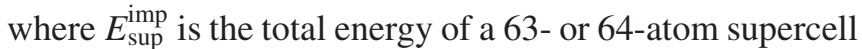
that contains the impurity, $E_{\text {sup }}^{\text {id }}$ is the total energy of a pure Ge unit cell (diamond structure, 2 atoms), $\mu_{\mathrm{Ge}}$ is the chemical potential of $\mathrm{Ge}$ (taken equal to the total energy
TABLE I. Heat of formation $\left(\Delta H_{f}\right)$ for the three impurity environments considered in this work, the relative displacements of the first nearest neighbor shell $(d)$ with respect to the starting configuration, and the calculated values for the isomer shift $\left(\delta_{0}\right)$ and the quadrupole splitting $\left(\Delta E_{Q, 0}\right)$ for Fe at $0 \mathrm{~K}$.

\begin{tabular}{|c|c|c|c|c|c|c|c|c|}
\hline & $\begin{array}{c}\mathrm{Fe} \\
\Delta H_{f} \\
(\mathrm{eV})\end{array}$ & $\begin{array}{c}d \\
(\%)\end{array}$ & $\begin{array}{c}\delta_{0} \\
(\mathrm{~mm} / \mathrm{s})\end{array}$ & $\begin{array}{c}\Delta E_{Q, 0} \\
(\mathrm{~mm} / \mathrm{s})\end{array}$ & $\begin{array}{c}\mathrm{Cu} \\
\Delta H_{f} \\
(\mathrm{eV})\end{array}$ & $\begin{array}{c}d \\
(\%)\end{array}$ & $\begin{array}{c}\mathrm{Ag} \\
\Delta H_{f} \\
(\mathrm{eV})\end{array}$ & $\begin{array}{c}d \\
(\%)\end{array}$ \\
\hline$S$ & 1.90 & -6.7 & 0.06 & & 1.34 & -4.6 & 1.63 & +0.0 \\
\hline$T$ & 3.19 & +0.1 & 1.08 & & 1.75 & +1.7 & 2.10 & +4.8 \\
\hline$S+V$ & 3.85 & -19.8 & 0.55 & 0.81 & 3.10 & -13.2 & 2.53 & -11.0 \\
\hline
\end{tabular}

per atom in bulk Ge), $n$ is the number of Ge atoms in the ideal 64-atom supercell that are replaced by either vacancies or impurities $(n=1,2)$ and $\mu_{\text {imp }}$ is the chemical potential of the impurity with respect to the elemental solid (ferromagnetic bcc-Fe, fcc-Cu, fcc-Ag). For all elemental solids, the lattice constant was optimized and then fixed for the 64-atom cells, but all atoms in those supercells were allowed to move to their equilibrium positions. The calculations were done by the APW + lo method within Density Functional Theory, as implemented in the WIEN2K code $[18,19]$. The Perdew-Burke-Ernzerhof [20] exchangecorrelation functional was used, the $k$-space sampling was done on a $4 \times 4 \times 4$ mesh in the 64 -atom cell, and a basis set corresponding to $K_{\max }=3.5$ a.u. was taken. The influence of the size of the supercell (up to 256 atoms) as well as the influence of the magnetic state of the $\mathrm{Fe}$ impurities on the calculations were verified and found to be small. After relaxation of the configuration with the impurities on the $S$ and the $T$ site, small displacements were found (Table I). A slightly reduced distance to the first nearest-neighbor $(d)$ shell has been calculated in the case of the substitutional impurity, while the impurity on the $T$ site pushes the neighbors slightly away. This is in agreement with previous calculations for $\mathrm{Fe}$ in $\mathrm{Ge}[10,11]$. Adding a vacancy to the substitutional impurity $(S+V)$ induces a large force on the TMs along the $\langle 111\rangle$-direction, resulting in the impurity ending up on the ideal sixfold coordinated $B C$ site with the vacancy split on the nearest neighbor positions: the split-vacancy configuration $[12,13]$. As can be seen from Table I, the nearest neighbor distance in this configuration is considerably reduced.

By comparing the heats of formation in Table I, it is clear that the three studied TMs prefer the $S$ site to the $T$ or $B C$ site. At first sight, this contradicts the experimental observation of the $B C$ site. The following arguments show this is not true, however. We calculated the heat of formation for a single neutral vacancy in Ge to be $2.23 \mathrm{eV}$ (in agreement with Ref. [21]). The total energy needed to have an impurity on the $S$ site and an isolated vacancy is larger than the heat of formation of the $B C$ configuration for all three TMs. Therefore, $S$ site impurities will trap the abundantly available mobile vacancies created during implantation, 
and-as the structural relaxation process in our calculations shows-will spontaneously evolve into the $B C$ site. Our results strengthen the hypothesis that the Fe atoms found on the $T$ site in Mössbauer experiments [8] are mainly produced by the $40 \mathrm{eV}$ recoil energy received during the $\beta$-decay of the ${ }^{57} \mathrm{Mn}$ atoms. This recoil energy is high enough to overcome the energy barrier between $S$ and $T$, but not enough to get reimplanted. $A b$ initio values for the isomer shift and electric-field gradient of nonmagnetic Fe on the $S, T$, and $B C$ sites (Table I) are in agreement with Mössbauer experiments [8], and suggest that an unidentified $B C$ site has been encountered before in such experiments. The $\mathrm{Fe}_{i}-V$ complex, with $\mathrm{Fe}$ on the $T$ site, as postulated in Ref. [8], would lead to $\delta=0.67 \mathrm{~mm} / \mathrm{s}$ and $\Delta E_{Q}=1.01 \mathrm{~mm} / \mathrm{s}$, in worse agreement with experiment. Reanalysis of the spectra from an earlier ECexperiment on $\mathrm{Cu}$ [9] indicates a large fit improvement by adding a $B C$ fraction to the $S$ fraction and only minor improvement after allowing displacements to the site halfway between the $S$ and $B C$ site, in accordance with our results.

In conclusion, we found direct experimental evidence that the ion implanted transition metals $\mathrm{Fe}, \mathrm{Cu}$, and $\mathrm{Ag}$ do not solely occupy the substitutional site, but the bondcentered site as well. This result contributes significantly to the understanding of the electrical properties of transition metals in germanium, since they are known to be electrically active on the $S$ site, while no active defect levels have been attributed to TMs on the $B C$ site. Corroborated by theory, this $B C$ fraction is attributed to impurity-vacancy complexes in the split-vacancy configuration. By investigating the heat of formation of this complex, it can be concluded that the mobile vacancies, created during the ion implantation process, will be trapped by substitutional impurities, resulting in the spontaneous occupation of the $B C$ site. Hence, this $B C$ behavior is a direct consequence of the presence of mobile vacancies, which were, in this specific study, created during the ion implantation process. However, these results are more generally valid since a wide variety of technologically important treatments are known to produce a large number of vacancies, such as the growth of Ge-films on Si [3], sputter deposition of metals on a Ge surface [22], and annealing of those deposited films to form germanides as Ohmic or Schottky contacts [23]. Furthermore, by intentionally introducing vacancies, e.g., with electron irradiation, we have presented an approach to relocate the TMs from the electrically active $S$ site to the $B C$ site.

This work was supported by FWO Flanders (G.0501.07 and G.0636.08), the K. U. Leuven projects GOA/2009/006 and INPAC EF/2005/005, the IUAP P6/42 program, the Portuguese Foundation for Science and Technology (POCI-FP-81921-2007) and the ISOLDE collaboration. S. D. acknowledges financial support from FWO.
[1] R. Hull and J.C. Bean, Germanium Silicon: Physics and Materials, Semiconductors and Semimetals (Academic, San Diego, 1999).

[2] Y. J. Yang, W. S. Ho, C. F. Huang, S. T. Chang, and C. W. Liu, Appl. Phys. Lett. 91, 102103 (2007).

[3] C. Claeys and E. Simoen, Germanium-based Technologies: From Materials to Devices (Elsevier, Amsterdam, 2007).

[4] P. Clauws and E. Simoen, Mater. Sci. Semicond. Process. 9, 546 (2006).

[5] P. Clauws, J. Van Gheluwe, J. Lauwaert, E. Simoen, J. Vanhellemont, M. Meuris, and A. Theuwis, Physica B (Amsterdam) 401-402, 188 (2007).

[6] E. Simoen, K. Opsomer, C. Claeys, K. Maex, C. Detavernier, R. L. Van Meirhaeghe, and P. Clauws, J. Appl. Phys. 104, 023705 (2008).

[7] P. Schwalbach, M. Hartick, M. Ciani, E. Kankeleit, B. Keck, R. Sieleman, B. Stahl, and L. Wende, Hyperfine Interact. 70, 1121 (1992).

[8] H. P. Gunnlaugsson, G. Weyer, M. Dietrich, M. Fanciulli, K. Bharuth-Ram, and R. Sielemann (ISOLDE Collaboration), Physica B (Amsterdam) 340-342, 537 (2003).

[9] U. Wahl, J. G. Correia, and J. C. Soares (ISOLDE Collaboration), Physica B (Amsterdam) 340, 799 (2003).

[10] A. Continenza, G. Profeta, and S. Picozzi, Phys. Rev. B 73, 035212 (2006).

[11] Z.Z. Zhang, B. Partoens, K. Chang, and F. M. Peeters, Phys. Rev. B 77, 155201 (2008).

[12] H. Höhler, N. Atodiresei, K. Schroeder, R. Zeller, and P. H. Dederichs, Phys. Rev. B 71, 035212 (2005).

[13] J. Coutinho, S. Öberg, V. J. B. Torres, M. Barroso, R. Jones, and P. R. Briddon, Phys. Rev. B 73, 235213 (2006).

[14] U. Wahl et al., Nucl. Instrum. Methods Phys. Res., Sect. A 524, 245 (2004).

[15] H. Hofsäss and G. Lindner, Phys. Rep. 201, 121 (1991).

[16] U. Wahl, Phys. Rep. 280, 145 (1997).

[17] H. Haesslein, R. Sielemann, and C. Zistl, Phys. Rev. Lett. 80, 2626 (1998).

[18] S. Cottenier (Instituut voor Kern- en Stralingsfysica, KULeuven, Belgium, 2002), (freely available from http://www.wien2k.at/reg_user/textbooks), ISBN 90807215-1-4.

[19] P. Blaha, K. Schwarz, G. Madsen, D. Kvasnicka, and J. Luitz, Wien2K: An Augmented Plane Wave Plus Local Orbitals Program for Calculating Crystal Properties (Karlheinz Schwarz, Techn. Universität Wien, Austria, 2001), ISBN 3-9501031-1-2.

[20] J. P. Perdew, K. Burke, and M. Ernzerhof, Phys. Rev. Lett. 77, 3865 (1996).

[21] A. Fazzio, A. Janotti, A. J. R. da Silva, and R. Mota, Phys. Rev. B 61, R2401 (2000).

[22] E. Simoen, K. Opsomer, C. Claeys, K. Maex, C. Detavernier, R. L. Van Meirhaeghe, and P. Clauws, Appl. Phys. Lett. 89, 202114 (2006).

[23] E. Simoen, K. Opsomer, C. Claeys, K. Maex, C. Detavernier, R. L. Van Meirhaeghe, and P. Clauws, J. Electrochem. Soc. 154, H857 (2007). 